\title{
A Novel Frameshift Mutation of SLC26A4 in a Korean Family With Nonsyndromic Hearing Loss and Enlarged Vestibular Aqueduct
}

\author{
Borum Sagong ${ }^{1} \cdot$ Jeong-In Baek ${ }^{2}$ Kyu-Yup Lee ${ }^{3}$ Un-Kyung Kim ${ }^{1,4}$ \\ ${ }^{I}$ Department of Biology, College of Natural Sciences, Kyungpook National University, Daegu; ${ }^{2}$ Department of Aroma Applied Industry, College of \\ Herbal Bio-Industry, Daegu Haany University, Gyeongsan; ${ }^{3}$ Department of Otorhinolaryngology-Head and Neck Surgery, Kyungpook National \\ University School of Medicine, Daegu; ${ }^{4}$ School of Life Sciences, BK21 Plus KNU Creative BioResearch Group, Kyungpook National University,
}

Daegu, Korea

Objectives. We aimed to identify the causative mutation for siblings in a Korean family with nonsyndromic hearing loss (HL) and enlarged vestibular aqueduct (EVA). The siblings were a 19-year-old female with bilateral profound HL and an 11-year-old male with bilateral moderately severe HL.

Methods. We extracted genomic DNA from blood samples of the siblings with HL, their parents, and 100 controls. We performed mutation analysis for SLC26A4 using direct sequencing.

Results. The two siblings were compound heterozygotes with the novel mutation p.I713LfsX8 and the previously described mutation p.H723R. Their parents had heterozygous mono-allelic mutations. Father had p.I713LfsX8 mutation as heterozygous, and mother had p.H723R mutation as heterozygous. However, novel mutation p.I713LfsX8 was not detected in 100 unrelated controls.

Conclusion. Both mutations identified in this study were located in the sulfate transporter and anti-sigma factor antagonist domain, the core region for membrane targeting of SulP/SLC26 anion transporters, which strongly suggests that failure in membrane trafficking by SLC26A4 is a direct cause of HL in this family. Our study could therefore provide a foundation for further investigations elucidating the SLC26A4-related mechanisms of HL.

Keywords. DFNB4; Hearing Loss; Enlarged Vestibular Aqueduct; SLC26A4; Novel Mutation

\section{INTRODUCTION}

Enlarged vestibular aqueduct (EVA; OMIM: 600791), the most abundant malformation of the inner ear, is often found by com-

- Received April 19, 2016

Revised May 22, 2016

Accepted May 26, 2016

- Corresponding author: Kyu-Yup Lee

Department of Otorhinolaryngology-Head and Neck Surgery, Kyungpook National University School of Medicine, 680 Gukchaebosang-ro, Jung-gu, Daegu 41944, Korea

Tel: +82-53-420-5781, Fax: +82-53-423-4524, E-mail: kylee@knu.ac.kr

- Co-Corresponding author: Un-Kyung Kim

Department of Biology, College of Natural Sciences, Kyungpook National University, 80 Daehak-ro, Buk-gu, Daegu 41566, Korea

Tel: +82-53-950-5353, Fax: +82-53-953-3066, E-mail: kimuk@knu.ac.kr puted tomography $(\mathrm{CT})$ in patients with sensorineural hearing loss (HL) [1]. Nonsyndromic HL with EVA is typically characterized by congenital, bilateral sensorineural HL, which can be progressive and usually ranges from severe to profound [2]. However, patients manifest EVA of various shapes and sizes, so the criteria for defining EVA differ depending on the size and measuring position [3,4]. Okamoto et al. [4] divided EVA cases into subgroups based on the shape and size of the vestibular aqueduct and tested for a relationship between the subgroups and HL characteristics or genotypes. However, differences in EVA manifestations did not correlate with HL levels or genotypes [4].

Mutations in SLC26A4 (OMIM: 605646) are among the most prevalent causes of EVA and are regarded the second most frequent cause of autosomal recessive nonsyndromic sensorineural

Copyright (C) 2017 by Korean Society of Otorhinolaryngology-Head and Neck Surgery.

This is an open-access article distributed under the terms of the Creative Commons Attribution Non-Commercial License (http://creativecommons.org/licenses/by-nc/4.0)

which permits unrestricted non-commercial use, distribution, and reproduction in any medium, provided the original work is properly cited. 
HL after mutations in GJB2 [5]. To date, more than $170 S L$ C26A4 mutations have been reported in diverse populations (Pendred/BOR Homepage: http://www.healthcare.uiowa.edu/ labs/pendredandbor/slcMutations.htm) as the cause of both nonsyndromic HL with EVA and Pendred syndrome (PS; OMIM: 274600) associated with other clinical findings, such as goiter, Mondini dysplasia, and incomplete iodide organification [6,7]. SLC26A4 comprises 21 exons and is situated on chromosome $7 \mathrm{q} 31$ [6]. Pendrin, the gene product of SLC26A4, is a 780 -amino acids hydrophobic membrane protein with 12 transmembrane domains [6,7]. Pendrin plays important roles in fluid and anion transport and maintenance of the endocochlear potential by mediating the exchange of bicarbonate, formate, chloride, and iodide ions [7]. Mutations in SLC26A4 can result in anion transport impairments in the thyroid and inner ear causing $\mathrm{HL}$ and/or goiter [8].

In this study, we describe a Korean family with $\mathrm{HL}$, including young siblings with sensorineural HL with EVA and compound heterozygous mutations in the SLC26A4 gene.

\section{MATERIALS AND METHODS}

\section{Subjects and clinical evaluations}

A Korean family with HL (KNUF01) was recruited from the Department of Otorhinolaryngology-Head and Neck Surgery at the Kyungpook National University Hospital in Daegu, Korea. Clinical evaluation of the family was conducted including the description of the family history, medical anamneses, and audiological testing for hearing level complemented by pure-tone audiometry (PTA) (Fig. 1A). We performed PTA with air-conduction at $500-8,000 \mathrm{~Hz}$ and computed the mean of thresholds checked at 500, 1,000, 2,000, and 4,000 Hz. The degree of $\mathrm{HL}$ was estimated based on PTA results as previously described [9]. Temporal bone CT was performed using a Somatom Sensation 16 (Siemens, Erlangen, Germany). One hundred unrelated Koreans who underwent PTA testing at the Kyungpook National University Hospital were recruited as normal controls. We obtained written informed consent from all participants, and this study was approved by the Institutional Review Board of the Kyungpook National University Hospital (KNUH BIO_09. 1007).

\section{H I G G H L L I}

- SLC26A4 mutations are the most prevalent cause of enlarged vestibular aqueduct (EVA).

- SLC26A4 mutations in a boy with EVA and his siblings were analyzed in comparison with 100 healthy controls.

- A novel heterozygous frameshift mutation p.I713LfsX8 was found in two siblings.

\section{Mutation analysis}

A FlexiGene DNA kit (Qiagen, Hilden, Germany) was used to extract genomic DNA from the peripheral blood of the KNUF01 family members and 100 normal controls. All 21 exons and the exon-intron boundaries of SLC26A4 (NM_000441.1) were analyzed by PCR using followed primer sets designed with the Primer3 software (http://primer3.ut.ee/) (Table 1), and their nucleotide sequences were analyzed using direct sequencing as described previously [10]. Mutation analysis was performed using a 3130xl Genetic Analyzer (Applied Biosystems, Foster City, CA, USA), Sequencing Analysis ver. 5.2 (Applied Biosystems), and Chromas Lite ver. 2.01 (Technelysium, Tewantin, QLD, Australia). The 1000 Genomes Project database (http://www.1000 genomes.org/) and the dbSNP database of the National Center for Biotechnology Information (NCBI; http://www.ncbi.nlm.nih. gov/) were used as references to assess the novelty of mutations found in this study. The potential pathogenicity of the novel mutation was predicted by MutationTaster (http://www.mutationtaster.org/), and multiple sequence alignments using CLC Sequence Viewer ver. 6.0.1 (CLC Bio, Aarhus, Denmark) were carried out to investigate the evolutionary conservation of the mutated amino acids.

\section{RESULTS}

\section{Clinical evaluation}

An 11-year-old boy (II-5) complained of HL and was examined for hearing level. His family (KNUF01) displayed autosomal recessive transmission (Fig. 1A). As the result of PTA, II-5 showed moderately severe HL with 70 and $73.3 \mathrm{~dB}$ average air-conduction in the right and left ears, respectively. As a result of periodic examination, he had revealed fluctuating $\mathrm{HL}$ at the age of seven. His sister (II-4), another affected family member, had exhibited profound bilateral HL from 5 years old. Other family members (I-1, I-2, and II-3) showed normal hearing. However, the affected sibling (II-1) was not available for study. Temporal bone CT of the proband showed Mondini dysplasia and bilateral EVA with width of the vestibular aqueduct greater than $1.5 \mathrm{~mm}$ [11] (Fig. 1B). Thyroid functional tests revealed normal serum concentrations of relevant substances: free thyroxine, $1.2 \mathrm{ng} / \mathrm{dL}$; triiodothyronine, $1.6 \mathrm{ng} / \mathrm{mL}$; thyroid-stimulating hormone, $2.32 \mu \mathrm{IU} /$ $\mathrm{mL}$; and antithyroglobulin antibody, $21.5 \mathrm{U} / \mathrm{mL}$. His neck ultrasonography produced normal results and did not reveal any pathologic lesions.

\section{Mutation analysis}

We identified two SLC26A4 variations in this family. The first variation was deletion of an adenine (c.2137delA) in exon 19 (left panels in Fig. 1C). This novel deletion was predicted to cause a frameshift and produce a truncated protein by a premature stop (p.I713LfsX8). Four members (I-1, II-3, II-4, and II-5) 


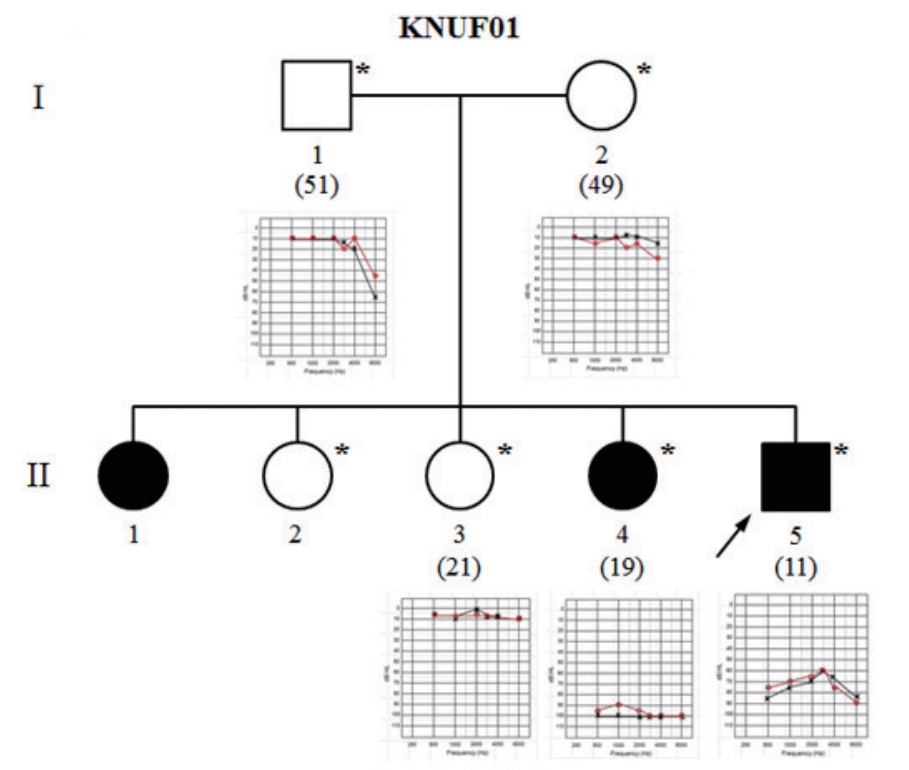

(A)
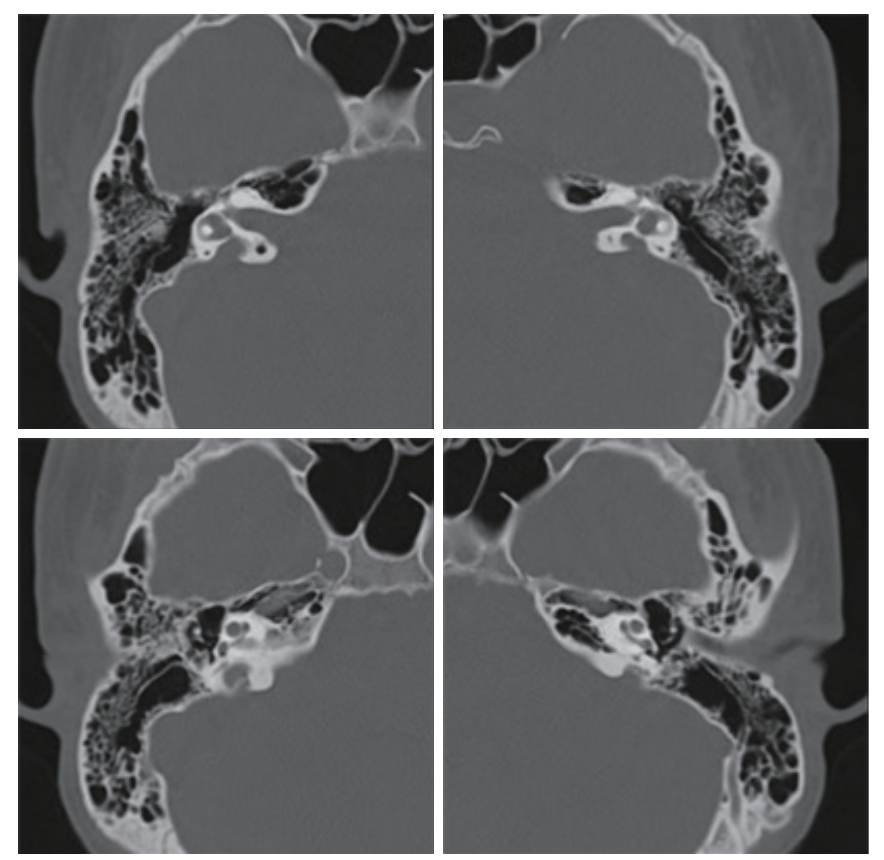

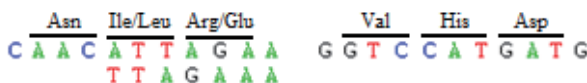

I-1
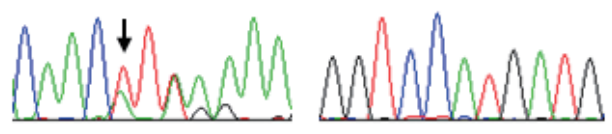

C Asn $\frac{\text { Ile }}{A \text { A C T T T A A A }} \frac{\text { Arg }}{\text { A }}$

G $\frac{V_{a l}}{G T C} \frac{H i s / \text { Arg }}{\text { C A T T }} \frac{\text { Asp }}{G \text { T G G }}$

$\mathrm{I}-2$
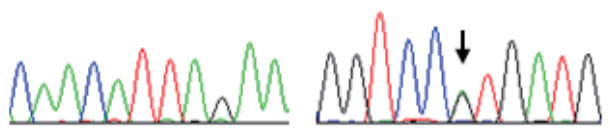

C Asn $\frac{\text { Ile }}{\stackrel{A}{A} \text { C }} \frac{\text { Arg }}{\stackrel{A}{A} \text { T A A }}$

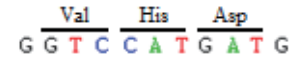

II-2

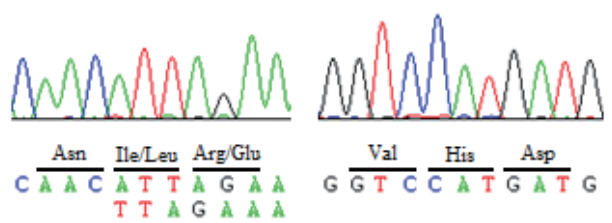

II-3
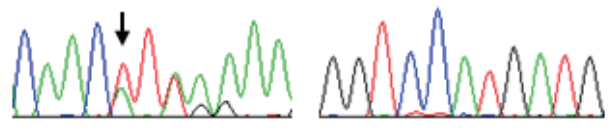

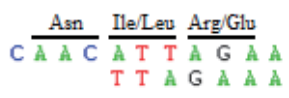

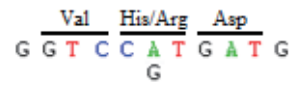

II-4
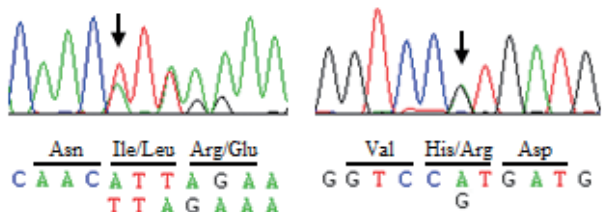

II-5
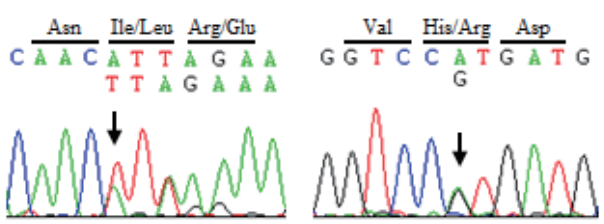

c. $2168 \mathrm{~A}>\mathrm{G}$

C

$\downarrow$ \&

[Homo_sapiens] IRKDTFFLTVHDA ILYLQNQVKSQEGQ [Rattus_norvegicus] IRKDRFFLTVHDA I LYLQNQAKSREGQ

[Mus_musculus] IRKDRFFLTVHDAILHLQNQVKSREGQ

[Xenopus_laevis] IKRD I FFLTVHDAVLH I EN LRKFYDGH

[Danio_rerio] VNRDLLFLSVHDA I LFIQHQNS I GYED

Consensus IRKDRFFLTVHDA I LXLQNQVKSREGQ

Conservation \begin{tabular}{c}
$100 \%$ \\
\hline$\square$
\end{tabular}

B

Fig. 1. Clinical and genetic characteristics of the Korean family with HL (KNUF01) family. (A) Pedigree and pure tone audiograms of the KNUF01 family. The two-generation pedigree including seven members is presented. Affected and unaffected individuals are indicated by shaded and clear symbols, respectively. The arrow indicates the proband, and asterisks indicate subjects of this study. Numbers in parenthesis refer to age. Red solid lines and black dashed lines of the pure tone audiograms indicate hearing thresholds of right and left ears, respectively. (B) The temporal bone computed tomograms of the proband (II-5). Right ear (left panels) and left ear (right panels) revealed enlarged vestibular aqueducts and Mondini dysplasia. (C) Verification of the c.2137delA and c.2168A $>$ G mutations by Sanger sequencing. The right and left panels provide the DNA sequences of SLC26A4 presenting the c.2137delA and c.2168A $>$ G variations in family members. Arrows indicate the changed bases. (D) Comparison of the human pendrin sequence with that of other species. Black and white arrows represent amino acid positions 720 and 723 of human pendrin, respectively. 
Table 1. Specific primer sequences of SLC26A4

\begin{tabular}{|c|c|c|c|}
\hline Exon & Forward primer & Reverse primer & Product size (bp) \\
\hline 1 & GACCACGGACCTCTTCCT & GGGGCAGAACTTTCAGCTTAT & 576 \\
\hline 2 & GGCTGCAGCTAACAGGTGAT & TTCCCAGGTAAGTTCATTTCG & 497 \\
\hline 3 & GTTCTTGGCAAAAGCATGGTA & TGAAGGGTAAGCAACCATCTG & 409 \\
\hline 4 & GGCTATCCTACCTGAAATGTGC & AAATGGAGTCAAATTGATCACG & 750 \\
\hline 5 & GCTCAGCTTCTTTCGTGAACA & GGATTCCCGTATATTTCCCTTC & 599 \\
\hline 6 & AGGACCGAAAGCCACATAAA & CTGGCCCAGACTCAGAGAAT & 527 \\
\hline $7-8$ & ATGGTTTTCATGTGGGAAGA & ACGGGCTGCTITAAACAAAT & 651 \\
\hline 9 & GAGGACAAAGAAATCAGCCAGT & GCAAACACAAGAGGAAAACCA & 506 \\
\hline 10 & CCCCAAGTACCTATCACGGTAA & ATTCAGGTGAGGGAGTGGAA & 431 \\
\hline $11-12$ & TTGTTCAGTTITGTGGCTTGA & CACAAAATGCAGTTCACATGG & 677 \\
\hline 13 & TGTAATTTGTTTGTGGATCATTG & GGAAGCTCAGAGTGTGTTGTTCT & 267 \\
\hline 14 & CGATTCCACACAAACACCAG & AGAAGGTGCACTGGATCAAAA & 411 \\
\hline 15 & CTGCGCAACAGAGTGAAACT & TCTCATTGCCCTACACAAAGG & 403 \\
\hline 16 & TTTTTGGCAGGATAGCTCAAG & CAGCGCTGCTGATITATTTC & 413 \\
\hline 17 & AAAGTTTGGGCTGAGGTGAAA & AAGTGGTGAAGCCAGTATCTCC & 585 \\
\hline 18 & TCCATGGTTTTGCAATAATAACC & GAACTCCTGCTTAGTTCCTTGG & 405 \\
\hline 19 & GTAGGGTGTGCCCTGTAGTC & GCATTATAGCTAATGCCCACTT & 628 \\
\hline 20 & CAATACAGCTGAAGAGGATTCTGA & GGGACAAAAGATCCCCAAGTA & 416 \\
\hline 21 & GCAACAGTGAGTGAGATTCAGTCTCC & GCCTGGTTCTGTAGCTTTAGGTA & 859 \\
\hline
\end{tabular}

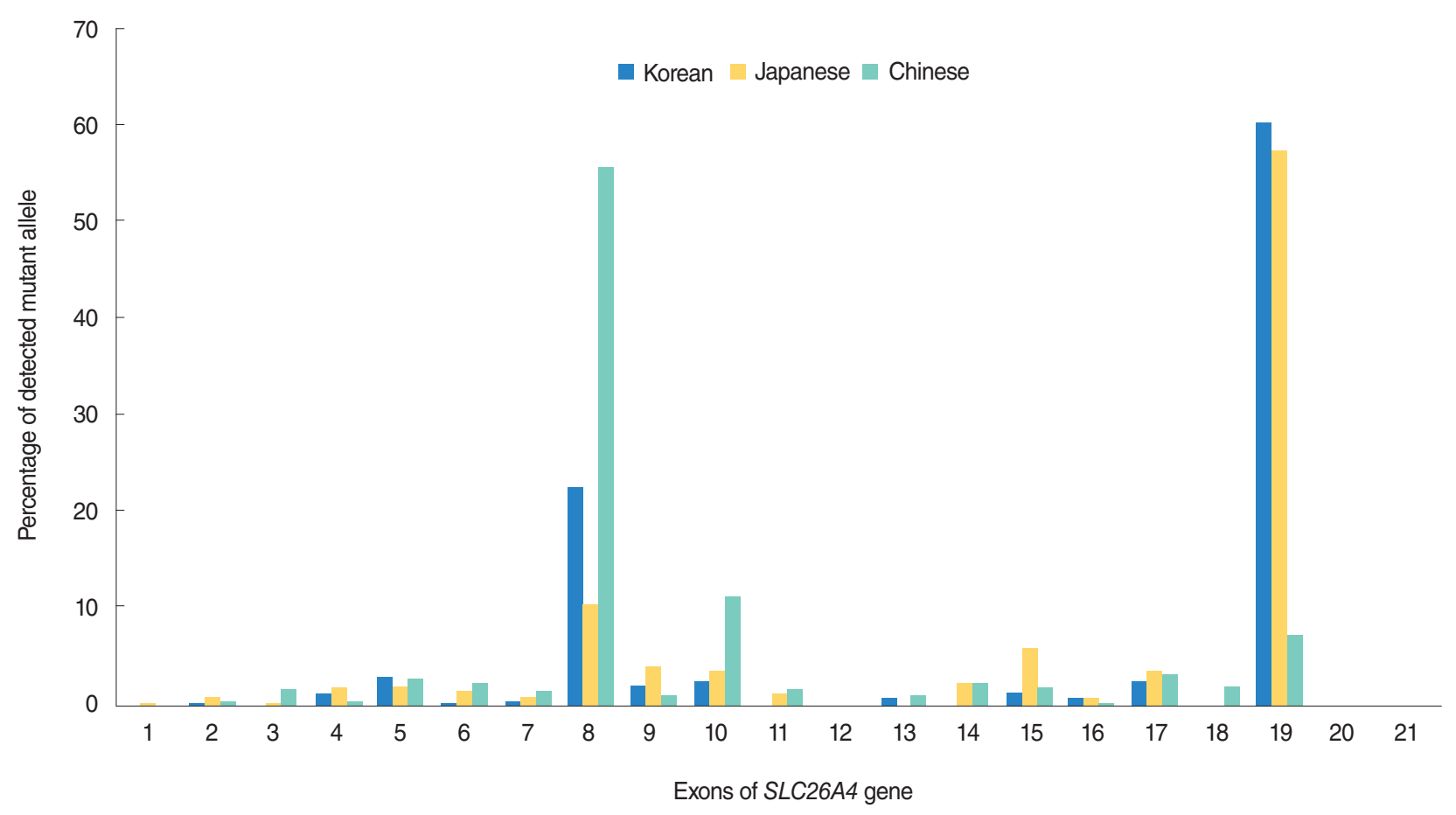

Fig. 2. Exonic distribution of SLC26A4 mutant alleles in East Asian patients with enlarged vestibular aqueduct (EVA). Total estimates for the Korean population are derived from four previous studies and in-house data [13-16]. The Japanese and Chinese population data were obtained from three and four previous studies, respectively [12,17-22].

of the KNUF01 family were heterozygous for this variation. However, it was not detected in two other family members (I-2 and II-2) or in 100 unrelated controls. One member (II-1) of this family was not available for this study. This variation was not reported in the NCBI dbSNP or 1000 Genomes Project databases, and truncated region caused by this variation was located in a highly conserved region among mammals (Fig. 1D). In addition, MutationTaster predicted this variation to be disease causing.

The second mutation was an adenine to guanine substitution (c.2168A > G) in exon 19 (right panels in Fig. 1C), which leads to substitution of histidine by arginine (p.H723R). Three members (I-2, II-4, and II-5) of the KNUF01 family were heterozy- 
gous for this mutation. This variation was present in the NCBI dbSNP and 1000 Genomes Project databases with a minor allele frequency (MAF) of $0.04 \%$ (rs121908362). In addition, this variation was previously reported to cause PS or nonsyndromic HL with EVA. The affected members (II-4 and II-5) of the KNUF01 family were heterozygous for both p.I713LfsX8 and p.H723R mutations. This finding indicates that the novel p. I713LfsX8 variation is the disease-causing mutation in this family as a compound heterozygous mutation with p.H723R.

\section{DISCUSSION}

Compound heterozygous patients with two SLC26A4 mutant alleles comprised up to $50 \%$ of Korean and Chinese patients and $70 \%$ of Japanese patients with HL $[12,13]$. We also identified compound heterozygous mutations with p.H723R and p.I713LfsX8 in two individuals in a family. These mutations were both located in exon 19 of SLC26A4. This exon is a mutation hotspot in East Asian populations and is particularly frequently mutated in the Korean population (Fig. 2)[12-22]. Among previously described mutant alleles in exon 19, the p.H723R mutation identified in this study was the most common mutation among Korean and Japanese patients with HL $[12-15,17,18]$. Yoon et al. [23] reported that the pendrin produced with this mutation was localized in endoplasmic reticulum tube-like structures and lacked $\mathrm{Cl}^{-} / \mathrm{HCO}_{3}{ }^{-}$exchange activity, whereas wild-type proteins reached the plasma membrane.

The other mutation, p.I713LfsX8, was reported for the first time in this study. This mutation led to early translational termination at amino acid position 720 in the sulfate transporter and anti-sigma factor antagonist (STAS) domain, which also modified the putative protein kinase A (PKA) binding site. The PKA site and the distal part of the STAS domain play an important role in regulating pendrin localization to the plasma membrane. Bizhanova et al. [24] reported that phosphorylation of the putative PKA site is not an absolute prerequisite for pendrin targeting to the plasma membrane. However, the STAS domain included in members of the SLC26A family regulates the stability, trafficking, and anion transport function of SLC26 family proteins $[7,25]$. The structural significance of this domain has been substantiated by the disease-causing nature of mutations therein among SLC26A family proteins [25]. Therefore, it is possible that the novel mutation discovered in our study impairs the function of pendrin by inactivating the distal STAS domain. Additionally, early stop codon causes nonsense-mediated mRNA decay (NMD) and nonsense mRNAs are rapidly decayed [26]. Therefore, the concentration of truncated protein would be extremely low in vivo. Even if the truncated protein is expressed, loss of the important functional domains will decide the abnormal levels of proteins. Because the patient had both p.I713LfsX8 and p.H723R mutations in each allele, another full-length proteins carrying the p.H723R mutation are also abnormal. Therefore, the patient cannot have any normal pendrin proteins.

Mutations in SLC26A4 have been reported to be responsible for a wide phenotypic spectrum of hearing problems, from typical PS to nonsyndromic HL with EVA [1,6]. There have been many reports documenting genotype-phenotype correlations in patients with mutations in SLC26A4. Several studies reported that patients with bi-allelic SLC26A4 mutations showed more severe HL, larger malformations of the inner ear, and more pronounced goiter than patients with no or mono-allelic mutations $[27,28]$. On the other hand, other studies suggested that there was no correlation between HL and SLC26A4 mutations $[19,29]$. The patient with bi-allelic mutations examined in this study showed profound HL but no evidence of goiter.

In conclusion, we describe here a case of nonsyndromic $\mathrm{HL}$ with EVA in young siblings of a Korean family, whose members harbored mutations in SLC26A4. We found compound heterozygous mutation of the SLC26A4 gene including p.I713LfsX8, a novel frameshift mutation. Thus, our study could provide a foundation for future investigation of the mechanisms of SLC26A4associated HL.

\section{CONFLICT OF INTEREST}

No potential conflict of interest relevant to this article was reported.

\section{ACKNOWLEDGMENTS}

This work was supported by the National Research Foundation (NRF) of Korea, funded by the Ministry of Science, Information and Communications Technology (ICT) and Future Planning (2015R1A2A2A01003438). This research was supported by a grant from the Korea Health Technology R\&D Project through the Korea Health Industry Development Institute (KHIDI), funded by the Ministry of Health \& Welfare, Republic of Korea (HI14C2119).

\section{REFERENCES}

1. Usami S, Abe S, Weston MD, Shinkawa H,Van Camp G, Kimberling WJ. Non-syndromic hearing loss associated with enlarged vestibular aqueduct is caused by PDS mutations. Hum Genet. 1999 Feb;104 (2):188-92.

2. Pique LM, Brennan ML, Davidson CJ, Schaefer F, Greinwald J Jr, Schrijver I. Mutation analysis of the SLC26A4, FOXI1 and KCNJ10 genes in individuals with congenital hearing loss. PeerJ. 2014 May;2: e384.

3. Madden C, Halsted M, Benton C, Greinwald J, Choo D. Enlarged vestibular aqueduct syndrome in the pediatric population. Otol Neurotol. 2003 Jul;24(4):625-32. 
4. Okamoto Y, Mutai H, Nakano A, Arimoto Y, SugiuchiT, Masuda S, et al. Subgroups of enlarged vestibular aqueduct in relation to SLC26A4 mutations and hearing loss. Laryngoscope. 2014 Apr;124(4): E134-40.

5. Hilgert N, Smith RJ, Van Camp G. Forty-six genes causing nonsyndromic hearing impairment: which ones should be analyzed in DNA diagnostics? Mutat Res. 2009 Mar-Jun;681(2-3):189-96.

6. Everett LA, Glaser B, Beck JC, Idol JR, Buchs A, Heyman M, et al. Pendred syndrome is caused by mutations in a putative sulphate transporter gene (PDS). Nat Genet. 1997 Dec;17(4):411-22.

7. Kopp P, Pesce L, Solis-S JC. Pendred syndrome and iodide transport in the thyroid.Trends Endocrinol Metab. 2008 Sep;19(7):260-8.

8. Bizhanova A, Kopp P. Genetics and phenomics of Pendred syndrome. Mol Cell Endocrinol. 2010 Jun;322(1-2):83-90.

9. Lee KY, Choi SY, Bae JW, Kim S, Chung KW, Drayna D, et al. Molecular analysis of the GJB2, GJB6 and SLC26A4 genes in Korean deafness patients. Int J Pediatr Otorhinolaryngol. 2008 Sep;72(9): 1301-9.

10. Sagong B, Seo YJ, Lee HJ, Kim MJ, Kim UK, Moon IS. A mutation of the succinate dehydrogenase B gene in a Korean family with paraganglioma. Fam Cancer. 2016 Feb 1 [Epub]. http://dx.doi.org/10. 1007/s10689-016-9874-8.

11. Cremers CW, Admiraal RJ, Huygen PL, Bolder C, Everett LA, Joosten FB, et al. Progressive hearing loss, hypoplasia of the cochlea and widened vestibular aqueducts are very common features in Pendred's syndrome. Int J Pediatr Otorhinolaryngol. 1998 Oct;45(2): 113-23.

12. Tsukamoto K, Suzuki H, Harada D, Namba A, Abe S, Usami S. Distribution and frequencies of PDS (SLC26A4) mutations in Pendred syndrome and nonsyndromic hearing loss associated with enlarged vestibular aqueduct: a unique spectrum of mutations in Japanese. Eur J Hum Genet. 2003 Dec;11(12):916-22.

13. Shin JW, Lee SC, Lee HK, Park HJ. Genetic Screening of GJB2 and SLC26A4 in Korean Cochlear Implantees: experience of Soree Ear Clinic. Clin Exp Otorhinolaryngol. 2012 Apr;5 Suppl 1:S10-3.

14. Lee HJ, Jung J, Shin JW, Song MH, Kim SH, Lee JH, et al. Correlation between genotype and phenotype in patients with bi-allelic SLC26A4 mutations. Clin Genet. 2014 Sep;86(3):270-5.

15. Song MH, Shin JW, Park HJ, Lee KA, Kim Y, Kim UK, et al. Intrafamilial phenotypic variability in families with biallelic SLC26A4 mutations. Laryngoscope. 2014 May;124(5):E194-202.

16. Park HJ, Hahn SH, Chun YM, Park K, Kim HN. Connexin26 mutations associated with nonsyndromic hearing loss. Laryngoscope. 2000 Sep;110(9):1535-8.

17. Suzuki H, Oshima A, Tsukamoto K, Abe S, Kumakawa K, Nagai K, et al. Clinical characteristics and genotype-phenotype correlation of hearing loss patients with SLC26A4 mutations. Acta Otolaryngol. 2007 Dec;127(12):1292-7.

18. Miyagawa M, Nishio SY, Usami S; Deafness Gene Study Consortium. Mutation spectrum and genotype-phenotype correlation of hearing loss patients caused by SLC26A4 mutations in the Japanese: a large cohort study. J Hum Genet. 2014 May;59(5):262-8.

19. Chai Y, Huang Z, Tao Z, Li X, Li L, Li Y, et al. Molecular etiology of hearing impairment associated with nonsyndromic enlarged vestibular aqueduct in East China. Am J Med GenetA. 2013 Sep;161A(9): 2226-33.

20. Chen K, Zong L, Liu M, Wang X, ZhouW, Zhan Y, et al. Developing regional genetic counseling for southern Chinese with nonsyndromic hearing impairment: a unique mutational spectrum. JTransl Med. 2014 Mar;12:64.

21. Hu H,Wu L, Feng Y, Pan Q, Long Z, Li J, et al. Molecular analysis of hearing loss associated with enlarged vestibular aqueduct in the mainland Chinese: a unique SLC26A4 mutation spectrum. J Hum Genet. 2007;52(6):492-7.

22. Wang QJ, Zhao YL, Rao SQ, Guo YF, Yuan H, Zong L, et al.A distinct spectrum of SLC26A4 mutations in patients with enlarged vestibular aqueduct in China. Clin Genet. 2007 Sep;72(3):245-54.

23. Yoon JS, Park HJ, Yoo SY, Namkung W, Jo MJ, Koo SK, et al. Heterogeneity in the processing defect of SLC26A4 mutants. J Med Genet. 2008 Jul;45(7):411-9.

24. Bizhanova A, Chew TL, Khuon S, Kopp P. Analysis of cellular localization and function of carboxy-terminal mutants of pendrin. Cell Physiol Biochem. 2011;28(3):423-34.

25. Sharma AK, Rigby AC, Alper SL. STAS domain structure and function. Cell Physiol Biochem. 2011;28(3):407-22.

26. Frischmeyer PA, Dietz HC. Nonsense-mediated mRNA decay in health and disease. Hum Mol Genet. 1999;8(10):1893-900.

27. King KA, Choi BY, Zalewski C, Madeo AC, Manichaikul A, Pryor SP, et al. SLC26A4 genotype, but not cochlear radiologic structure, is correlated with hearing loss in ears with an enlarged vestibular aqueduct. Laryngoscope. 2010 Feb;120(2):384-9.

28. Ito T, Choi BY, King KA, Zalewski CK, Muskett J, Chattaraj P, et al. SLC26A4 genotypes and phenotypes associated with enlargement of the vestibular aqueduct. Cell Physiol Biochem. 2011;28(3):54552.

29. Pera A, Dossena S, Rodighiero S, Gandia M, Botta G, Meyer G, et al. Functional assessment of allelic variants in the SLC26A4 gene involved in Pendred syndrome and nonsyndromic EVA. Proc Natl Acad Sci U SA. 2008 Nov;105(47):18608-13. 